\title{
The New Forms of Medicines Producing in Oncology
}

\section{Shoikhedbrod $M^{*}$}

Electromagnetic Impulse Inc., Toronto, Ontario, Canada

\begin{abstract}
The existing methods of chemotherapeutic treatment do not permit to calculate the precise dose of medicine and the time of its action on the tumor that leads to the unpredictability of result, and, therefore, to the ineffectiveness of the use of treatment itself. Furthermore, the damage of normal cells on the chemotherapy becomes the reason for a whole series of the complications, connected with side-effects action of the chemotherapy. The paper presents the developed mechanism of processing of the fundamentally new form of anti-tumorigenic medicines with the calculated precise dose of the action of cytostatic on the tumor cells at the moment of their mitosis (indirect division of cells).

The new form of anti-tumorigenic medicines is a support material with the equally distributed spherical drops of cytostatic. The support material, thus, is a solid at room temperature and is melted at human body temperature, during its implanting into the body parts of patient, which supply the tumor by the blood, will dosed act directly on tumor cells at the moment of their mitosis using the equally distributed drops of cytostatic.

Any treating chemotherapist can calculate impact dose and time of the action of cytostatic that fall accurately on the mitosis of cancerous cells if he knows the volume of each drop and their total quantity in the support material, and also the time of melting of the support material. The calculated dose of cytostatic in the support material is reached by the injection of the assigned by chemotherapist volume of the cytostatic in the form of the spherical drops through the specific gap inside the camera for the molten support material by way of bubbling in the weightlessness conditions.
\end{abstract}

Keywords: Anti-tumorigenic medicines; Tumor cells; Mitosis; Bubbling; Weightlessness conditions

\section{Introduction}

For the tumor cells is characteristically the fast growth and relatively fast dividing. The anti-tumorigenic medicines (cytostatics), which are used in the chemotherapy, not only block these processes, but also cause irreversible injury and loss of such cells [1-5]. Many normal cells also rapidly grow and are divided. Their number includes: the cell of bone marrow, mucous membrane of the cavity of mouth and gastrointestinal tract, reproductive system, hair follicles. Therefore anti-tumorigenic medicines cause the damage also of these normal cells that also is the reason for a whole series of the complications, connected with the sideeffects action of chemotherapy. There are several methods of delivering the medicine to the organism in the chemotherapy. Standard of them is the intravenous therapy, whose basic purpose is completely release of the patient from remained cancerous cells, which began to strike the tissues of the healthy organs [1].

However, with this method through the vein the medicines fall into the blood and strike both the tumor and healthy cells of entire organism. In this case it is not possible to calculate the precise dose of medicine and the time of its action on the tumor, which leads to the unpredictability of result, and, therefore, to the ineffectiveness of the use of chemotheraputic action. Another method of delivering the medicine to the organism in the chemotherapy is the regional chemotherapy, which is defined as the local chemotherapy, which influences on the part or area of body or organ [6-9]. In this case the medicine is entered to the artery, which falls through the blood directly to the tumor or its area. With the first passage of medicine through the tumor in the tissue the large number of cytostatic is sucked due to the regional therapy. Immediately after this therapy the blood has to be washed via chemo filtering, in the course of which the surplus of medicine is moved away. Thus, the chance of the entry of medicine to the tumor is in a considerable degree increased and is decreased appearance among the patients of side effects.
However, just as in the first case, in this case it is not possible to calculate the precise dose of medicine and the time of its action on the tumor, which leads to the unpredictability of result, and, therefore, to the ineffectiveness of the use of chemotherapeutic action. Thus, the existing methods of chemotherapy cannot be acknowledged effective and their results predicted. The damage of normal cells with the chemotherapy becomes the reason for a whole series of the complications, connected with the side-effects action of chemotherapy. The number of appearances of such complications includes: nausea and vomiting, the pitting of the mucous membrane of oral cavity, baldness, anemia, the increased fatigue.

The side-effects action of chemotherapy can explain also the high probability of bleedings and infectious complications. During the process of treatment can appear such undesirable consequence as the disturbances of the function of kidneys, the bladder, nervous system and other organs. The necessity of the development of the new forms of medicines and new methods of their delivery to the tumor, capable of effectively destroying tumor cells, without affecting the healthy cells of organism, is appeared. The paper presents the developed mechanism of processing the fundamentally new form of anti-tumorigenic medicines with calculated precise dose of the action of cytostatic on the tumor cells at the moment of their mitosis (indirect division of cells).

*Corresponding author: Shoikhedbrod M, Electromagnetic Impulse Inc., Toronto, Ontario, Canada, Tel: 6509065; E-mail: mshoikh@primus.ca

Received December 11, 2017; Accepted December 18, 2017; Published December 26, 2017

Citation: Shoikhedbrod M (2017) The New Forms of Medicines Producing in Oncology. J Blood Lymph 8: 194. doi:10.4172/2165-7831.1000194

Copyright: (c) 2017 Shoikhedbrod M. This is an open-access article distributed under the terms of the Creative Commons Attribution License, which permits unrestricted use, distribution, and reproduction in any medium, provided the original author and source are credited. 
The new form of anti-tumorigenic medicine is support material with the equally distributed spherical drops of cytostatic. Knowledge the volume of each drop and their total quantity in the support material, and also the time of melting of the support material permits to any treating chemotherapist to calculate impact dose and time of the action of cytostatic directly in the time of the mitosis of cancerous cells.

\section{Materials}

As the support material can be used on the lipophilic basis the cocoa butter, its alloys with paraffin and hydrogenizes fats, vegetable and animal hydrogenizes fats, solid fat, lanolin (also spermaceti), the alloys of the hydrogenizes fats with wax, solid paraffin and other bases, permitted for the medical practice. As the support material also can be used on the hydrophilic basis gelatinglycerol gels, the alloys of polyethylene oxides with different molecular weights and other substances, permitted for the medical practice. The gelatin glycerol basis is made from the medical gelatin, glycerin and water.

Thus, the support material is a solid material at room temperature and melts at a body temperature and will with the precise dose act on tumor cells during implanting of produced medicine to the parts of body, which supply blood of the tumor using the equally distributed drops of cytostatic. Knowledge of the volume of each drop and their total quantity in the support material, and also of the time of melting of the support material permits to any treating chemotherapist to calculate impact dose and time of the action of cytostatic directly in the time of the mitosis of cancerous cells.

\section{Method and Developed Devices}

The basis of the developed method of processing of the new form of anti-tumorigenic medicines included the use of an exceptional special feature of weightlessness-isotropism i.e., the identity of physical properties in all directions. The developed method [10] uses the isotropism for processing the support material with equally distributed spherical drops of cytostatic in the conditions of microgravity. The volume and quantity of drops of cytostatic are assigned according to previously calculated data of chemotherapists, which determine impact dose and time of implanting medicine to the parts of body of patient, which supply blood of the tumor.

The time of melting of support material was determined by the standard time of melting its lipophilic or hydrophilic basis. The method of processing of new forms of anti-tumorigenic medicines in the weightlessness conditions included the processing of support material on the lipophilic or hydrophilic bases with the uniform distribution of the spherical drops of cytostatic of calculated volume. The uniform distribution of spherical drops of cytostatics of calculated volume, in entire volume of support material is achieved in the weightlessness conditions, because of the unique property of weightlessnessisotropism.

The calculated dose of cytostatic in the support material is reached by the injection of the assigned by the chemotherapist volume of the cytostatic through the specific gap in the special constructed ampule camera with molten support material. The process of transmission into the molten support material of the drops of dense liquid (cytostatics) by the dispersion of this dense liquid (cytostatics) through the small holes, in our case - gap in the special constructed ampule camera with molten support material, is called bubbling.

For evaluation of the diameter of formed in the molten support material of the spherical drop of cytostatic is used the formula:

\section{$d=\left(6 d_{0} \sigma / \Delta \rho g\right)^{1 / 3}$}

Where $\boldsymbol{d}_{\boldsymbol{0}}$-diameter of gap, $\boldsymbol{\sigma}$-the surface tension of the molten of the support material, $\Delta \rho$-difference of the densities of the molten support material and cytostatic. Then the volume of the drop of cytostatic is simply calculated using the formula:

$$
V=(4 / 3) \pi(d / 2)^{3}
$$

Where $V$-the volume of the drop of the cytostatic, $d$-the diameter of the drop of cytostatic, which is determined by the formula (1), $\pi=3.1416$.

If entire volume of the injected cytostatic is determined as $V_{1}$, then possible to calculate the number of drops of cytostatic, and consequently the dose of all spherical drops of cytostatic, equally distributed in entire volume of the support material by formula:

$$
n=V_{1} / V
$$

Using the eqns. (1-3) any chemotherapist will be able to prepare the calculated dose of cytostatic for the anti-tumorigenic medicine dosed action on the tumor cells in the period of their mitosis.

It should be noted that the melting of anti-tumorigenic medicine in the patient body is produced gradually from the periphery to the center of medicine with equally quantity of dose of the action of cytostatic on the tumor. For the realization of the developed method of processing of the new form of anti-tumorigenic medicines with equally distributed spherical drops of cytostatic of support material was developed the special set "PCSFW" (physical chemistry studies of the special features of weightlessness) as part of the regular set "PION" (CSRIM) for further use of entire complex on the board of the International Space Station.

On the set "PCSFW" were installed the special constructed ampules for producing the anti-tumorigenic medicine with the uniform distribution of the drops of cytostatic. The construction of ampule for producing the anti-tumorigenic medicine with the uniform distribution of the drops of cytostatic (Figure 1) included glass camera from molybdenum glass -1 for the melting of support material, which contains thermocouple -6 for maintaining the necessary temperature in the camera, connected through the gap -7 with the camera -4 , filled by calculated dose of cytostatic. All working parts of the ampule are "dressed" in the plastic jacket (2) for the isolation of the taking place in the cameras processes with the purpose of safety of the operatorcosmonauts and the space station.

From above and from below the camera for the melting of support material were arranged by the heating elements in the form of tablets (3). Camera was supplied by the piston (5) for the injection via the bubbling of the spherical drops of cytostatic into the molten support material. The construction of ampule provides easy replacement of glass camera with the produced anti-tumorigenic medicine on the new glass camera for processing the new anti-tumorigenic medicine. As support material were used on the lipophilic basis the cocoa butter or on the hydrophilic basis the gelatin glycerol gels. The temperature of warming was not exceeding $37^{\circ} \mathrm{C}$. Temperature distribution inside the molten support material is registered by the chromel-copel thermocouple, entered in the middle of glass camera with the molten of support material.

\section{Results}

Ground tests of the developed method were conducted within the framework of the program of the physical chemistry research of the behavior of multicomponent systems in the weightlessness conditions on the base of CSRIM (Central Scientific Research Institute of Machine 
building) and short-term microgravity tests were conducted on the board of the flying laboratory IL-76K. One of the purposes of program was determination the possibility of using the inertial force for processing the equally saturated by spherical drops of cytostatic of the support material in the weightlessness conditions.

For the realization of this purpose of program was developed the special set "PCSFW" (physical chemistry studies of the special features of weightlessness) as part of the regular set "PION" (CSRIM) for further use of entire complex on the board of the International Space Station. On the set "PCSFW" were installed the special constructed ampules (Figure 1) for producing the anti-tumorigenic medicine with the uniform distribution of the drops of cytostatic.

Processing of the anti-tumorigenic medicine is carried by the following actions of operator-cosmonaut:

1. The preparation of "PCSFW" set to the work:

- The remote control panel of set was docked with the platform of set through the socket;

- The platform was connected to the power supply and video measuring of set "PION";

- The ampule was inserted and fixed on the platform;

- The heating elements of ampule were connected to the platform through the socket "heating" and the thermocouple of ampule was connected to the terminal "thermocouple".

2. The anti-tumorigenic medicine processing:

- The power supply was turned on the remote control panel (presence of electric power supply in the network was indicated by work of indicator light);

- The glass camera of ampule was preheated by the tumbler of heating turned on;

- With the melting of model material, video measuring of set "PION" switched on;

- By pushing of start knob on the remote control panel was powered the electromagnetic mechanism trigger of the piston, which injected through the gap between the cameras via the

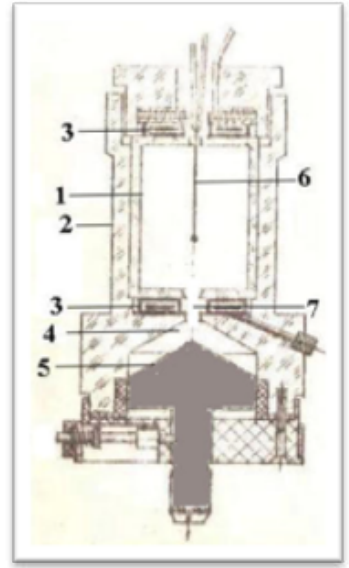

Figure 1: The construction of ampule for producing the antitumorigenic medicine with the uniform distribution of the drops of cytostatic. bubbling of the spherical drops of cytostatic into the molten support material;

- The tumbler of heating was turned off;

- After the solidification of the producing anti-tumorigenic medicine, the video measuring of set "PION" was turned off.

3. Dismantling of "PCSFW" set:

The dismantling of "PCSFW" set was achieved in the reverse order of the preparation of "PCSFW" set to the work.

\section{Conclusion}

The obtained results of conducted ground and short-term microgravity tests permitted to establish the fundamental possibility of processing of the anti-tumorigenic medicine with the uniform distribution of the spherical drops of cytostatic. During the test the precalculated dose of cytostatic spherical drops in the support material was reached by the injection of the assigned by the chemotherapist volume of the cytostatic through the specific gap by bubbling to the special constructed ampule camera with molten support material.

The tests have proved the uniform distribution of spherical drops of cytostatics of calculated volume in entire volume of support material in the weightlessness conditions. The short-term microgravity conditions $(20-30 \mathrm{sec})$ have not permitted to produce the solid anti-tumorigenic medicine due to the lack of time for solidification.

It is appropriate to use the developed method and devices in the space station microgravity conditions for commercial processing of the anti-tumorigenic medicine with the uniform distribution of the spherical drops of cytostatic inside. Thus, the developed method of processing of the anti-tumorigenic medicine with the uniform distribution of the spherical drops of cytostatic inside, allows during implanting of medicine into the body parts of patient, which supply the tumor by the blood, with the calculated dose of cytostatic to act directly on tumor cells at the moment of their mitosis for their effective destruction, without affecting the healthy cells of organism.

\section{References}

1. Machak GN (2003) Modern chemotherapy of the localized form of osteosarcoma. Vestnik Ronts 14

2. Verma R, Foster R, Horgan K, Mounsey K, Nixon H, et al. (2016) Lymphocyte depletion and repopulation after chemotherapy for primary breast cancer. Breast Cancer Research 18: 10.

3. Mir O, Ropert S, Goldwasser F (2009) Cisplatin as a cornerstone of modern chemotherapy. The Lancet Oncology 10: 304

4. Verrill M (2009) Chemotherapy for early-stage breast cancer: a brief history. British Journal of Cancer 101: S2-S5

5. Schuell B, Gruenberger T, Kornek G, Dworan N, Depisch D, et al. (2005) Side effects during chemotherapy predict tumour response in advanced colorectal cancer. British Journal of Cancer 93: 744-748.

6. Ishikawa O, Ohigashi H, Imaoka S, Sasaki Y, Kameyama M, et al. (1997) Regional chemotherapy to prevent hepatic metastasis after resection of pancreatic cancer. Hepatogastroenterology 44: 1541-1546.

7. Arybzhanov DT, Saburov AR (2009) Regional chemotherapy in the preoperation treatment of patients by cancer of stomach, Siberian oncologic periodical.

8. Gantsev Sh, Arybzhanov DT, Saburov AR (2017) The results of treating the patients by cancer of stomach with the use of regional chemotherapy and transarterial chemoembolization metastases in the liver. New formation 1: 61-66.

9. Balch C, Urist M, McGregor M (1983) Continuous regional chemotherapy 
for metastatic colorectal cancer using a totally implantable infusion pump: A feasibility study in 50 patients. The American Journal of Surgery 145: 285-290.
10. Shoikhedbrod MP (2017) Computer modeling and the new technologies in oncology. Lambert Academic publishing: Toronto. 